\title{
L'ACTION DE LA GELÉE ROYALE SUR LES ANIMAUX SUPÉRIEURS ET SUR L'HOMVE CRITIQUE DE LA REVUE DES JOHANSSON
}

\author{
PAR
}

\section{R. CHA UVIN}

Station de recherches apicoles, Bures-sur-Yvette.

Depuis un grand nombre d'années, la gelée royale est trop souvent l'objet d'une publicité excessive qui gêne considérablement la recherche et tend à discréditer le produit. De nombreux hommes de science et médecins ont émis à cet égard des appréciations péjoratives et justifiées. Cependant, négligeant les propagandes trop intéressées, chercheurs et cliniciens cherchaient à établir ou à préciser les qualités d'une drogue qui a bouleversé en France l'apiculture de nombreuses régions et dont la vente a permis à de nombreux apiculteurs un rééquipement indispensable. Le sujet n'est plus neuf, puisque les Johansson (I958) dénombrent une centaine de publications scientifiques qui traitent de la gelée sous divers aspects, et dont plusieurs sont très récentes. J'en ai parlé moi-même à plusieurs reprises (Chauvin, I956) et il ne peut être question de reprendre sous forme abrégée, la revue fort étendue que les Johassson y consacrent dans les numéros d'octobre et novembre $195^{8}$ du "Bee World".

Mais on peut faire à cette revue de graves reproches. Les auteurs semblent vouloir démontrer, à toute force, que la gelée n'a d'action véritable ni sur les mammifères, ni sur l'homme ; or, beaucoup de chercheurs et de cliniciens sont maintenant d'un avis contraire. Il m'apparaît que l'analyse que les Johansson font de leurs travaux n'en reflète pas exactement les conclusions. Je voudrais tenter de rétablir ici une interprétation plus correcte des résultats déjà publiés. On me trouvera peut-être à mon tour partial quant à l'action re la gelée; j'avoue y être encouragé par plusieurs travaux en cours dans mon propre laboratoire et dans divers laboratoires étrangers, et qui sont en cours d'achèvement ou de publication. 


\section{I. - L'action sur les Mammifères.}

I,es Johansson usent d'un procédé typographique très clair si toutefois on 1'utilise sérieusement; c'est-à-dire qu'ils présentent les résultats en colonnes; par exemple, dans l'énumération des travaux, une colonne est réservée au nombre des témoins, une autre au nombre des traités; si le texte analysé ne porte pas d'indications claires là-dessus, on place un " ? " ou u11 " $O$ » à la colonne " témoins " par exemple, ce que 1e lecteur trop pressé interprétera comme une absence de témoins. Par exemple, mon travail de I956 avec HÉBERT (C.R. Soc. Biol.) porte un point d'interrogation à la colonne " témoins "; cependant l'existence de témoins est explicite page 333 (ligne $n^{\circ}$ I ; lignes $29,32,34$ ). Je dois préciser qu'il s'agit d'un travail d'histochimie où il est absolument indispensable de comparer chaque animal traité à un animal témoin. Cela est tellement évident pour un histochimiste qui connait en particulier 1a disposition extrêmenent irrégulière des phosphatases de la corticosurrénale (dont il s'agissait), que je n'avais pas éprouvé le besoin de le noter en détail. On trotuve également un "? " à la colonne "dosage " alors que la dose de GR employée est précisée page 3.32 (ligne $n^{0} \mathrm{II}$ ). Qu'il me soit permis de regretter aussi la mention " no effect on growth" (pas d'effet sur la croissance) en regard de la citation du même travail, alors qu'il n'y est pas question de la "croissance ", mais seulement du poids des organes!

A propos de l'action hyperglycémiante de la gelée (Chauvin, 1956) si les Johansson consentent à mentionner dans leur première colonne le fait que mes expériences ont porté sur 530 souris (ce qui leur donne tout de même une base statistique assez confortable), ils inscrivent à nonveau "? ? " dans la colonne " traités » et " témoins ». Iit il est vrai que je n'ai pas pris soin dans la note à l'Académie des Ściences sur l'hyperglycémie induite chez les souris par la GR, de mentionner le nombre de traités et le nombre de témoins : les JoHansson ne savent probablement pas que les règles imposées à un texte par l'Académie sont très strictes et (qu'il faut tout y condenser, parfois à l'excès. Ils conviendront peutêtre avec moi qu'il est difficile de supposer qu'un biologiste effectue plus de 500 glycémies sans réserver des témoins; et qu'alors il est inutile et incorrect de suggérer le contraire par certaines dispositions typographiques. 'Tous les biochimistes savent d'ailleurs que la glycémie des rats et des souris est excessivement variable, même à jeun, dans des conditions aussi standard que possible, et qu'il est rigoureusement impossible d'essayer un agent hyperglycémiant sans réserver un nombre égal de témoins. Je vois bien que j'ai eu tort de ne pas préciser un certain nombre d'évidences... Notons en passant que les JoHansson ne connaissent que le travail de Chauvin et HéBERT paru dans les Comptes-Rendus 
de la Société de Biologie en 1956 et qu'il ignorent le mémoire de Biologica Latina (vol. IX, I956) beaucoup plus développé.

Je me montrerai plus sévère pour l'interprétation des travaux d'ARDRY, présentée aussi sous forme de tableau. Dans la subdivision de ce tablean intitulée "circulation ", il est indiqué qu'ARDRY aurait travaillé sur un lapin seulement, sans réserver de témoins (dans ses études sur la production d'anticorps et la pression sanguine chez le lapin soumis à l'action de la (GR); et ceci laisse planer évidemment un doute grave sur le sérieux de ses travaux.

Or, il ressort très clairement du texte d'ARDRY (paragr. : Études inmunologiques, I $^{\text {er ligne; }}$ premier mémoire dans les Annales pharmaceutiques Françaises, 1956, t. I4) que des mesures ont été pratiquées sur " des " lapins; ce qui a dû tromper les Johansson est l'expression de la page précédente, dernier paragraphe " examen du sérum de lapin " où lapin est employé au singulier, tournure de la langue française qui signifie simplement "le lapin en général "; les Johansson semblent en avoir déduit qu'il s'agissait d'un seul lapin! Les mêmes auteurs signalent aussi l'absence de témoins dans les travaux d'ARDRY portant sur la numération globulaire du lapin traité à la gelée royale. Que ne tiennent-ils compte du tableau III de ARIRY (mémoire présenté à l'Académie de Pharmacie le Ier février I956) où l'auteur donne les chiffres des érythrocytes et lencocytes pour chaque lapin, avant et après le traitement à la gelée royale? A moins que les JoHANsson ne veuillent dire qu'ARDry n'a pas étudié d'autres séries de Iapins auxquels on aurait injecté dans le même temps une substance banale telle que la caséine, ou quoi que ce soit d'autre. A cela on peut répondre qu'il est difficile d'imaginer quelle pourrait être une bonne substance de contrôle quand on étudie la gelée ; plusieurs chercheurs américains qui m'ont entretenu du problème semblent considérer que la caséine ou l'albumine feraient fort bien l'affaire ; c'est oublier bien vite la complexité de la gelée oì se trouvent par exemple des sucres et des lipides en proportion importante. Pourquoi alors ne faire qu'tu1 contrôle à la caséine ? P'ourquoi n'ell pas imaginer avec différentes espèces de lipides et de sucres ou avec des mélanges de caséine, de lipides et de sucres? En réalité de tels contrôles sont pratiquement dénués de sens en présence d'une substance aussi complexe que la gelée où les éléments actifs sont par trop indéterminés. Je pense donc qu'au point de vue "stratégique ", la première close à faire devant une substance inconnue est d'opérer comme l'a fait ARDRY en comparant les sujets à eux-mêmes; on voit ainsi rapidement si le résultat est banal et facilement interprétable, ou s'il donne prise au contraire à une analyse plus poussée. Et dans un second temps, il convient d'opérer, à mon sens, non pas tant avec des substances témoins dont le " contrôle " n'est qu'illusoire à ce stade, mais avec des fractionnement de plus en plus poussés. 
Pout en terminer avec l'analyse du travail d'ARDRy telle que la comprend Johansson, on remarque dans la subdivision du tableau intitulée "adrenals" (surrénales) "I2 témoins, I2 traités" (cobayes). Or, il semble évident, d'après les tableaux II et III d'ARDry (Mém. présenté à l'Académie de Pharmacie le I"r fér. 1956) que l'auteur a travaillé su' 25 cobayes en en sacrifiant trois par jour, pour doser séparénent l'acide ascorbique dans les surrénales de chaque cobaye. Une série de I 2 individus recevait une seule injection de (KR (avec trois témoins au début); nut autre série de 9 recevait deux injections à 7 jours d'intervalle. I'expérience était donc agencée d'une toute antre façon que ne le décrit Johissssox.

\section{Les tests cliniques tels qu'ils ont até publiés et tels que les déerivent les Johansson.}

Pour ne pas donner des dimensions trop considérables à cette revue. je ne puis analyser de la même façon les autres essais pratiqués par différents auteurs, et la manière dont les rapportent les Johansson. I a valeur de ces travanx est bien entendu inégale; mais les inexactitudes multiples déjà relevées dans la revue des Jontasssox ne m’encouragent guère à détailler une telle analyse.

Je regrette de dire que la partie de la revue des Jonansson consacrée aux essais cliniques me paraît la plus critiquable. Ici, les auteurs placent en italique dans leur tableau les conclusions qui, à leur sens, " ne prêtent pas à me mesure objective, ou sont présentées sans données à l'appui, ou deviennent ininterprétables par suite d'autres traitements effectués en même temps. "I isons tout de suite que la quasi-totalité des conclusions du tableau sont en italictue, à deux ou trois exceptions près! Quelques exemples sont nécessaires pour montrer la " liberté d'interprétation " des références. Ians le travail de MAIOSSI et (irandr (I946) que les auteurs présentent eux-mèmes comme préliminaire, les observations méritent d'être considérées avec soin, car elles portent sur des enfants prématurés en état de dystrophie grave. Iòn général, le médecin. très inquiet, essaie sur ces enfants une foule de drogues plus ou nonons efficaces dans l'espoir d'éviter la cachexie finale. Particulièrement intéressant est le cas d'un prénaturé à 7 mois traité sans interruption depuis le premier mois par des amino-acides, des protéolysats, des vitanines, etc. sans résultat. Son asthénie ne cesse qu'arec l'administration orale de gelée royale, et la courbe de poids ne remonte qu'à ce moment. Dans les cas $11^{\circ} 2$ et 3 , des jumeaux prématurés ont été traités dans les I 5 premiers jours de leur vie de la même façon que dans le cas $11^{\circ}$ I, sans résultat; le traitement est interrompu, on y substitue de la gelée royale : à partir de ce moment précis, la courbe de poids remonte régulièrement. D'autres 
patients, cités par MAIossr et GRANin ont effectivement reçu en même temps que la GR, d'autres traitement (des antibiotiques, à cause d'accidents infectieux) qui rendent l'interprétation difficile; pourquoi les JoHANsson ne retiennent-ils que ces derniers cas dans leur tableau?

PROSI'ERI et RAGAzZINI (I956) ont travaillé sur des nourrissons souffrant d'hypotrophies à étiologie variable (naladie cceliaque, stéatorrhée, sécquelles de maladies infectieuses, etc.). Toute thérapentique a été suspendue pendant l'administration de la gelée royale. S'il se produisait chez certains sujets 111 accident infectienx, on cessait la CrR, et on ne la redonnait qu'après avoir supprimé les antibiotiques. Or l'angnentation de poids correspondant à l'administration de GR a été très marquée, ainsi que l'augmentation du nombre des globules rouges et de l'hémoglobine; lat protidémie s'est normalisée ainsi que le rapport albunine/globuline.

SARROUY et ses collaborateurs $(1956)$ travaillant eux aussi sur des hypotrophiques, sont particulièrement nets dans leurs conclusions et leurs cas $1^{\circ} \mathrm{I}, 4$, 6 et 7 me paraissent très démonstratifs. I)ans ces cas, les traitements nombreux qu'instaure dans son angoisse le praticien sont restés sans effet. Mais seulement après l'administration de gelée royale l'appétit est devenu meilleur, et le poids a augmenté "l'action sur l'état général le tonus et surtout l'appétit parait devoir être signaléc... Ia gelée nous parait un adjuvant utile sinon déterminant dans les hypotrophies graves du nourrisson. "

Or toutes ces conchusions sont rejetées en bloc par les Jomansson puisqu'ils les mettent en italique. Je serais curieux de savoir si une reprise de poids chez un nourrisson hypotrophique ne leur parait pas objective, et comment il se fait qu'une série de traitements jusqu'à présent inefficaces ne deviennent tout à coup décisifs que lorsqu'on leur substitue la gelée?

A vrai dire ce problème de la reprise de poids préoccupe effectivement les Johussson, puisque (p. 277 du bee Iforld) ils lui cherchent une explication. Dans des pays à zoncs sous dércloppées comme l'Italie ou l'Algérie, disent-ils, la nourriture au sein au-delà du $\sigma^{\mathrm{e}}$ mois peut conduire à l'anémie et à différents troubles jusqu'à ce que le régime soit supplémenté. bes études sur des enfants carencés (11 Italie ont nontré que l'adjonction de vitanine $B_{12}$ (en closes plus fortes toutefois que ce qui se trouverait effectivement dans la gelée) provoque nne angmentation du poids, qui ne se produit pas sur des sujets bien nourris. Seulement, les sujets traités par les différents anteurs se tronvaient en dinique, et les JoHANsson admettront sans doute que le régime des cliniques curopéennes est convenablement surveillé et que pour des enfants hypotrophiques, il était aussi riche que possible? De plus, ils avaient été traités auparavant par une foule de drogues, y compris des vitamines. Je ne pense pas qu'il soit d'une bonne méthorle scientifique de chercher à tout prix n'importe 
quelle explication pour rejeter un phénomène que l'on a d'abord décidé do ne pas admettre.

Reste le très important travail de IDEsTrem (I34 cas) qui a porté sur deux catégories d'adultes, soit de grands vieillards présentant tous les stigmates de la sénescence à son dernier stade, soit des patients plus jeunes, souffrant de divers désordres neuro-psychiques. Les Jomansson placent encore en italiques toutes les conclusions de DESTRLin, sauf les 25-28 p. Ioo de cas que I)EsTrem lui-même reconnaît négatifs. Ainsi donc, les trois quarts des résultats positifs paraissent sans valeur aux JoHANSSON alors que seul le quart négatif leur semble digne d'être retenu? J'ajoute que pour le lecteur impartial le texte de DESTREM ainsi que les observations détaillées qu'il présente montrent l'étonnement d'un clinicien devant des malades chroniques (grabataires depuis des mois ou des années et habitués à prendre toute sorte de médicaments qui ne leur font rien) qui se relèvent brusquement après quelques injections de gelée. I,e fait intéressant est constitué, ne l'oublions pas, par la chronicité de leur mal, qui les "immunise» en quelque sorte, contre toute espèce de traitement, à moins qu'il ne soit réellement efficace.

Je me crois donc le droit de faire les plus grandes réserves sur une façon de présenter des documents qui en inverse le sens et détourne le lecteur de se reporter aux sources. I.es tableaux établis par les Johansson déforment les conclusions des auteurs cités, et on y voit beaucoup trop d'erreurs matérielles.

Passons maintenant à des objections plus sérieuses faites par les JOHANSSON : à savoir celle du "stress " et celle de l'absence de placebo dans les recherches cliniques.

\section{La gelée royale et lo stress.}

Pour mes lecteurs qui seraient plus familiers avec la science apicole qu'avec la médicale, je précise que la notion de "stress » ou d'agression est due à SErYE (I950) qui distingue plusieurs phases de réponse de l'organisme à une blessure, une émotion, ou toute autre agression physiologique : la phase d'alarme ou de shock se caractérise par l'hypothermie, l'hypotension, la dépression neuromusculaire, l'hémoconcentration, 1'hypochlorémie, 1'hyperalcaliémie, 1'hyperglycénie, la leucopénie, etc. I a phase de résistance correspond à une augmentation d'activité de la cortico-surrénale, avec tendance à l'involution des organes lymphogènes comme le thymus et la rate, et inversion de beaucoup de symptômes observés dans la première phase.

D'après JoHANsson, les phénomènes que j'ai observés avec HÉBER'T et ceux que décrit ARDRY ne sont ricn d'autre qu'un stress. J'ai d'ailleurs moi-même songé à cette hypothèse, et ARDRY ne la néglige pas non plus, 
et tente en plusieurs endroits de baser sur les théories de Sirry son interprétation des effets de la gelée royale. Renarquons toutefois que la description du stress par SEIYY est si large, ct englobe une si grande variété de réactions que l'on se demande quel est l'agent thérapentique ou antre qui n'est pas "stressant " par quelque endroit! Notons aussi que le sucre du sang par exemple, ne pent guère répondre que de trois façons à un agent quelconque : ou bien il diminue ou bien il augmente, ou il reste à son taux initial! Eit il en est de mêne de tous les constituants du sérum. si par dessus le marché, on imagine qu'une phase, comme celle d'agression est télescopéc, ou an contraire anormalement allongée on obtient tout ce qu'il faut pour faire rentrer n'importe quel phénomène (lans le lit de Procuste du stress. Mais les explications trop bien ajustées d'avance à tous les problènes s'écronlent tôt on tard après avoir stérilisé la recherche pendant un certain temps.

Si la mode, si puissante en physiologie humaine et surtont en médecine, vent introduire partout le stress, i1 ne s'ensuit pas que nous devions nous denander uniquement devant unc réaction si clle rentre ou 11011 dans la classification de Sintys. Il est plus utile de décrire avec soin le phénomène, sans rouloir le classer trop vite à l'aide d'une théorie ou d'une autre.

Or, que voyons-nous lorsque la (iR est introduite dans l'organisme d'un animal? Un certain nombre de symptômes qui rentrent assez bien dans la classification de SELYE : par exemple la déplétion de l'acide ascorbique des surrénales, l'action antagoniste de l'AC'TH sur les effets de la gelée (ARDRY) 1'hyperglycémie (CHAUVIN et H'́BER'T). Mais quand les Johansson, citent à l'appui de la théorie du stress l'involution des organes lymphoïdes décrite par CHirvin et HÉBkR' ils oublient que ces auteurs la considèrent comme très légère et non significative au point de vue statistique (Chavvin et HuBERT, in Biologica Latina, I956). De ménc, la déplétion des phosphatases dans la cortico-surrénale, identique à celle qui se produit après injection de cortisone, n'est pas si facile ¿̀ interpréter; non plus que la diminution des cétostéroïdes urinaires, alors qu'ils devraient augmenter (ARDRY). Ce qui montre bien que l'injection de gelée donne lieu à un stress assez spécial, si stress il y a. (Ajoutons que les mêmes phénomène's s'observent après injection de divers extraits lipidiques purifiés de gelée royale, qui en principe, ne donnent pas lieu à stress ?)

D'ailleurs d'autres expériences, dont JoHANSSON n'a pu avoir connaissance, car elles sont seulement en cours de publication, montrent que la gelée, ingérée par sondage xsophagien et non plus administrée par piqûre donne lieu à une hyperglycémie plus nette et plus constante que par injection (à des doses plus élevées à vrai dire). Classiquement, on adnet que les protéines administrées par la bouche ne donnent guère lieu à stress. 


\section{La question du placebo.}

Un reproche général fait par JoHANSSON à tous les essais cliniques, et qui mérite l'attention, est l'absence générale de placebo. En réalité, des placebos ont bien été utilisés dans les toutes premières expériences, mais elles n'ont pas été publiées et les Johansson ne pouvaient le savoir. Rappelons, pour les lecteurs peu familiers avec la phraséologie médicale, qu'un placebo doit présenter exactement l'aspect de la drogue en voie d'expérimentation, mais ne contenir que du lactose ou de l'amidon, en tout cas rien d'a priori actif. On utilise en général le placebo suivant la méthode dite " de la double ignorance " (double blind method) c'est-àdire que devant des comprimés identiques, par exemple, ni le malade ni le médecin ne savent où se trouve la série dont l'activité est présumée. Un troisième expérimentateur compulse ensuite les résultats, et, en s'aidant de l'analyse statistique, examine si la drogue présumée efficace a donné un résultat vraiment différent du placebo. I a méthode, utilisée depuis I8I a moins, n'a été vraiment comprise et appliquée en médecine clinique que depuis peu de temps: elle donne à l'expérimentation sur 1'homme la précision et l'objectivité dont elle manquait souvent.

Comme le remarquent Worf et Pinsky (I953) beaucoup de médicaments nouvellement introduits sont d'abord l'objet de commentaires élogieux de la part des médecins; puis au bout d'un certain temps, leur efficacité semble disparaître complètement. I'où l'on concluait que les auteurs des premiers rapports favorables avaient été le jouet d'une illusion. Mais cela parait maintenant moins évident: il suffit de supposer que 1'effet "placebo " qui s'amortit dès que la drogue n'apparaît plus comme une nouveauté, rend parfaitement compte des premiers résultats. Si bien que l'effet d'une drogue, suivant la frappante formule de BEECHer (I955) est égal à son effet propre, plus son effet de placebo.

Jusqu'oì peut aller l'action d'un placebo? KEA'TS, MOS'EILER et I ASAGNE (I953) l'examinent dans le cas de violentes douleurs postopératoires consécutives aux incisions: ils trouvent que parmi les drogues sédatives, la morphine ou la codéine n'ont pas plus d'effet qu'un placebo, mais que l'aspirine en a beaucoup plus; $30-35$ p. Ioo des individus sont en moyenne parfaitement soulagés par l'administration du seul placebo. La même conclusion est retrouvée par BEECHER (I955) travaillant sur I ooo patients souffrant de douleurs post-traumatiques, d'angine de poitrine, de céphalée, de nausée, ou même de grippe.

Il convient peut-être de tempérer ces conclusions par quelques observations de MODEL.. (I958). En laissant au malade le soin de porter lui-même sur une çarte le résultat de la cure (aspirine et placebo) et en traitant statistiquement les résultats globaux, on arrive à pronver que les patients 
sont incapables de distinguer entre différentes doses d'aspirine et entre l'aspirine et le placebo! Mais en en faisant prendre les observations par un médecin (toujours avec la méthode de la double ignorance évidemment) on arrive à montrer au contraire que les sujets distinguent parfaitement entre placebo et aspirine et entre différentes doses d'aspirine. I,es placebos ne dispensent pas de prendre toutes les précautions psychologiques d'usage dans la notation des expériences!

CLEGHORN et ses collaborateurs ont mis l'accent sur les manifestations objectives qui suivent 1'administration du placebo; que 1'on utilise 1'hormone hypophysaire AC'TH ou un placebo, on obtient les mêmes modifications sur le taux des neutrophiles, les lymphocytes et des éosinophiles, du potassium, du sodium, des I7-cétostérö̈des, des lipides réducteurs ou du rapport acide urique/créatinine.

Notons toutefois que d'après I,ASAGNA, MOSTELLFR et von I'ELSINGir, on observe une décroissance de l'action du placebo quand on l'administre plusieurs fois; mais cette décroissance peut être immédiate (dès la seconde dose) ou au contraire se faire attendre jusqu'à la quatrième ; elle est très irrégulière suivant les sujets, si bien que d'après BEECHFR, on ne peut jamais prédire après l'administration d'un prenier placebo, ce yui arrivera lorsqu'on donnera le second.

De cette très brève revue, il faut conclure évidemment que l'édifice clinique et thérapeutique élevé avant 1'emploi systématique des placebos ne s'écroule pas pour autant, mais que les médecins doivent apprentre à se défier d'une impression clinique, même appuyée d'examens objectifs, surtout si on ne la retronve que dans 30 p. Ioo des cas, c'est-idire précisément dans les limites d'action du placebo. Que dans le cas de malades chroniques inconscients (sénescents au dernier stade par exemple) déjà traités journellement par une foule de drogues, l'effet placebo est amoindri; et qu'il doit être bien faible chez des nourrissons!

Je crois donc pouvoir conclure que les observations de DESTREM auxquelles il vient de joindre de nombreuses autres, non publiées encore s'expliquent malaisément par le seul effet du placebo. Il obtient beaucoup plus que $35 \mathrm{p}$. Ioo de résultats favorables (ceux qu'ils classe comme bous ou très bons dépassent $69 \mathrm{p}$. Ioo!). Oublier ce dernier pourcentage et ne mettre en évidence que les 25 p. Ioo d'échecs et l'absence de placebo, comme le font les Johansson, c'est manquer, à mon sens, d'objectivité.

\section{La spécilicité d'action de la gelée royale.}

Cette dernière objection, que 1'on rencontre quelquefois dans la littérature, ne me parait pas non plus convaincante : les effets de la gelée ne seraient pas à retenir, parce qu'ils manquent de spécificité : la gelée 
ne guérit pas une maladie bien connue et nettement délimitée. Je remarque aussitôt qu'il en est de même d'une foule de drogues comme l'aspirine ou les tranquillisants; et que la linite entre la spécificité et la non-spécificité me paraît bien difficile à tracer.

\section{Résumí et conchusions.}

La revue générale présentée par les Johansson est inexacte en plusieurs points : les auteurs notent par exemple l'absence de témoins lorsque des témoins ont été effectivement réservés; attribuent à un travail des conclusions négatives sur la croissance alors qu'il y est question de tout autre chose, prétendent que l'augmentation du poids chez un uourrisson n'a pas de valeur objective, etc.

Si l'on considère impartialenent 1'ensemble des travaux de physiologie et de clinique effectués sur la gelée, on doit reconnaître qu'au cours de ces dernières années les physiologistes ont mis en évidence une action sur les animaux de laboratoire qui ne se ramène qu'en partie à un stress classique; et que d'autre part les cliniciens ont trouvé trois directions d'utilisation (hypotrophie du nourrisson, sénescence, anxiété). S'il est absolument évident qu'il ne s'agit encore que de travaux d'approche qui demandent à être précisés, il est injuste de prétendre plus longtemps que l'effet de la gelée sur les organismes supérieurs est seulement douteux et subjectif.

\section{Addendum.}

Bien que les quelques références qui vont être analysées maintenant n'aient pas de rapport direct avec ce qui précède, il m'a paru intéressant de faire connaitre différentes recherches qui ont été signalées au Congrès d'Apiculture de Rome, en septembre 1958 . Je remercie à cette occasion les professeuts Murano, Lenzi et Fal aschini, les Drs Mormone et Wiser, qui ont bien voulu me communiquer un compte-rendu détaillé de leurs travaux.

Au point de vue des essais sur les animaux de laboratoire, il faut signaler les recherches de Falaschini, qui ont porté sur $5 \mathrm{I}$ poussins $(25$ traités, 26 témoins) et sur 22 veaux. Ira gelée royale était administrée par la bouche à raison de $100 \mathrm{mg}$ par jour pour les poussins. L'auteur n'a observé qu'un léger retard de croissance par rapport aux témoins; retard que je considère comme intéressant, car je l'ai retrouvé moi-nême chez les souris traitées à la gelée (Chauvin, en cours de publication). Sur les veaux, l'Al,aschin n'a obtenu aucun résultat. L'examen histologique des organes (hypophyse, thyroïde, surrénales, testicules, ovaires) n'a fourni aucune donnée intéressante.

Je dois rapprocher ces résultats d'autres, également négatifs, qui m’ont été fournis par DELSOL, de la Faculté des Sciences cle Lyon. I1 a utilisé des injections aux poussins de broyats hypophysaires de souris traitées à la gelée. On prélève la thyroïde $\mathrm{d} u$ poussin trente heures après la première injection, et l'on mesure la hauteur de l'épithélium thyroïdien en prenant toutes précantions du point de vue statistique et biométrique. Les souris avaient reçu quatre injections de $20 \mathrm{mg}$ de gelée. I.eurs hypophyses n'ont produit aucun allonge- 
ment de l'épithélium thyroïdien des poussins, qui soit différent des résultats obtenus avec les hypophyses de souris témoins.

Au point de vue des essais cliniques, une contribution importante à mon sens est due aux cliniciens italiens: ils fournissent des tests physioksgiques de l'action de la gelée sur l'homme, alors que nous en étions jusqu'ici assez dépourvus (à part quelques observations d'ARDRY). I,EAzi confirme les résultats de Murano et MarTINETTI sur l'augmentation de la consommation d'oxygène après administration de roo à $200 \mathrm{mg}$ de gelée ; mais pas de modifications sanguines, et pas de crises réticulocytaires, comme certains l'ont dit. Nette augmentation de l'élimination des I7-cétostéroïdes et des II-oxycorticoïdes, ce qui tendrait à confirmer les résultats que l'on verra plus loin au sujet du test de THorN.

Mormone, Nunziata et Spina ont travaillé avec des extraits déprotéinés de gelée royale, en injections intraveineuses à des bébés dystrophiques. Ils ont noté des amélionations du poids, de la crase hématique, de l'appétit, de l'état général. Mais surtout, des modifications importantes de la glycémie capillaire et de la glycémie veineuse. La première augmente de $47 \mathrm{p}$. Ioo, I/2 heure après l'injection ; la seconde de $22 \mathrm{p}$. IOo une heure après. Le test de THORN est positif. La hausse du cholestérol du sérum est nette dans I I cas sur IS. MORMONE signale également l'amélioration des enfants dystrophiques traité: à la gelée, également par voie intraveineuse, et notamment l'amélioration des suppurations cutanées; la vitesse de sédimentation et le taux de glycogène des leucocytes subiraient aussi certaines modifications. Mormonf et NunziATA signalent de plus une augmentation de la protidémie "modeste, mais constante $\%$. La fraction des albumines a augmenté de $2,3 \%$ dans $60 \%$ des cas; la fraction des globulines diminue du même taux, dans $60 \%$ des cas. Mais ce sont les alphaglobulines qui diminuent alors que les bêtaglobulines augmentent (tout au moins chez les sujets où les taux initiaux étaient élevés). Les gammaglobulines diminuent chez les sujets à taux initial élevé, augmentent chez les sujets à taux initial paranormal.

WEBER enfin a administré à des enfants mongoliens, âgés de 3 à 12 mois, Ioo $\mathrm{mg}$ par jour de $\mathrm{G} \mathrm{R}$ (voie orale). Le développement mental s'accélère sensiblement, l'excitation motrice tend à régresser. D'après l'auteur de ces expériences, qui dirige une clinique de psychiatrie infantile à Wroclaw, les progrès des enfants mongoliens sous l'influence de la G. R. sont " incomparablement supérieurs " à ce qu'on obtient par d'autres moyens thérapeutiques (trarail non publié).

Toutes ces recherches, dont le nombre s'accroît rapidement, confirment semble-t-il, le point de vue exposé auparavant et qui rend optimiste quant à l'avenir de la gelée royale et à ses effets sur la physiologie humaine.

\section{SUMMARY}

Johansson's review on royal jelly effects countains several misinterpretations and material errors : for example, several times the authors state " 110 control " when in fact controls have been reserved; or " no effect on growth" quoting a paper where no attention was paid to growth; and so on.

After an impartial review of physiological and clinical work(whose value may be big or in some cases poor, as ever in biological research) one must recognize that in the last years physiologists found an action on laboratory animals 
of royal jelly not fully understandable in terms of stress only ; secondly, the clinicians found also three directions of investigation (hypotrophy of the young, senescence, anxiety). I fully agree in the idea that all this work is only an approach and need further and deeper investigation ; but it is unfair to say ever and again that royal jelly effects on higher organisms are only doubtful and subjective.

\section{RÉFÉRENCES BIBLIOGRAPHIQUES}

ARDRY (R.). - Contribution à l'étude de la gelée royale. I. II. III. $A n n$. Pharm. Franc., XIV, 97-II8, I956.

Brecher (H. K.). - The powerful placebo. Joum. Am. Med. Assoc., I 602-6, I955.

Johansson (T. S. K.), Johansson (M. P.). - Royal jelly II. Bee World, 39, $249-64 ; 277-86$, I958.

Chauvin (R.), Hebert (S.). - Effets des injections de gelée royale sur les Mammifères. C. R. Soc. Biol., CL, 332, I956.

Chauvin (R.), Hebert (S.). - Action de la gelée royale d'abeilles injectée aux Mammifères. Etude biologique et histologique. Biol. Latina, IX, $44 \mathrm{I}-52, \quad$ I956.

Chauvin (R.). - Sur un principe de la gelée royale d'abeilles, actif sur la glycémie des Mammifères. C. R. Acad. Sc., 243, I 920-I, I956.

I)ESTREM (H.). - Expérimentation sur la gelée royale d'abeilles en pratique gériâtrique (134 cas). Rev. lir. Géront., I3 p., I956.

Keats, Mosteller et Iasagna, in BEECHER (v. plus haut).

Marossi (C.), Grandi (F.). - O Osservazioni sulla gelatina reale nell'alimentazione degli immaturi (Nota preventiva). Atti I ${ }^{\circ}$ Convegno per 10 Studio dell'applicazione dei prodotti delle api nel campo medico-biologico (en abrégé Atti). Istituto Nazionale di Apicoltura, Bologna, aprile I956, p. 130 .

MODEL, (W.), HoUde (R. W.). - Factors influencing clinical evaluation of drugs. Journ. Am. Med. Assoc., 2, I90-99, 1956.

Mormone (V.), Nunziata (B.). - Sul1' impiego della gelatina reale per via parenterale in bambini distrofici. Atti V. Conv. Naz. Med., in Aggiornam. Pediatr. IX, IO-II, I958.

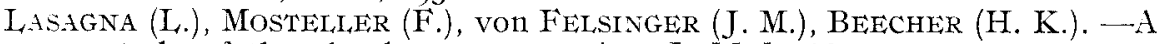
study of the placebo response. $A m$. J. Med., 16, 770-9, I954.

Prosperi (P.), Ragazzini (F.), Francalancia (L.). - Sull'impiego terapentico della pappa reale delle api negli stati di denutrizione della prima infanzia. (Atti, p. 135, I956).

SArrouy (C.) RAFir (A.), Leuteneger (M.). - Essai de traitement de huit cas d'hypotrophie grave du nourrisson par les extraits lyophilisés de gelée royale. Pédiatrie, 4, 1956.

WoLF (S.), PINSKY (R. H.). - Effects of placebo administration and occurrence of toxic reactions. J. Amer. Med. Assoc., 155, 339-4I, I954. 